\title{
Clinical Assessment of Mental Rotation in Hemiplegic Patients after Stroke
}

\author{
TAKASHI TANAKA, RPT ${ }^{1)}$, MINORU YAMADA, RPT, PhD ${ }^{2)}$, SHINOBU INAGAKI, PhD ${ }^{1)}$ \\ 1)Division of Health Sciences, Graduate School of Medicine, Osaka University: 1-7 Yamadaoka, Suita, \\ 565-0871 Osaka, Japan. \\ TEL: +81-06-6879-2585, FAX: +81-06-6879-2581, E-mail: t.tanaka@sahs.med.osaka-u.ac.jp \\ 2) Division of Human Health Sciences, Graduate School of Medicine, Kyoto University
}

\begin{abstract}
Purpose] In this study, we investigated the motor imagery ability of hemiplegic stroke patients. [Subjects] The subjects were 29 healthy individuals (average age, $63.5 \pm 11.7$ ), and 31 hemiplegic stroke patients (average age, $64.5 \pm 9.5)$. [Method] Motor imagery was evaluated using the reaction time of mental rotation in response to photographs of a rotated hand.[Results] The reaction time was delayed in stroke patients compared to healthy individuals, and post-stroke mental rotation was better when viewing photographs of the unaffected than of the affected side. Impaired attention is associated with mental rotation ability; in contrast, motor function, deep sensory factor, lapsed days from onset and the brain damaged side were less associated with rotation ability in stroke patients. [Conclusion] Therapeutic intervention using motor imagery is expected to have a high therapeutic effect because of its affect on impaired attention.
\end{abstract}

Key words: Hemiplegic patients, Mental rotation, Attention

(This article was submitted Jun. 8, 2010, and was accepted Jul. 14, 2010)

\section{INTRODUCTION}

Ischemic stroke is a devastating injury caused by interruption of the blood supply to regions of the brain. In humans, this type of injury strongly correlates with paralysis, sensory deficits, impairments in learning and memory, and disability in activities of daily living [ADL]; however, there are few effective therapies for the treatment of ischemic stroke ${ }^{1)}$. Recent evidence suggests that the use of motor imagery training, as an additional therapy, has an effect on motor recovery after stroke ${ }^{2-6)}$.

Motor imagery, which involves imagining actions without their execution, can be defined as an active process in which the representation of a specific action is internally reproduced within working memory but without any overt output ${ }^{7)}$. Significant lateral organization was observed during both the actual movements and motor imagery in the primary motor cortex, somatosensory cortex, supplementary motor area, premotor cortex, thalamus, and cerebellum ${ }^{8,9)}$; therefore, motor imagery training is expected to be a method of prompting motor learning and motor skills without voluntary movement.

Mental rotation of a body part is a simple method of measuring a person's capacity for motor imagery. Mental rotation is the ability to mentally identify an object, which is presented in a rotated manner ${ }^{10)}$. Previous studies have reported activity in the cerebral motor-related area measured by functional magnetic resonance imaging when subjects viewed a photograph of a rotated hand ${ }^{1-13)}$. This activity was the same as that observed during first-person motor imagery. This result suggests the possibility that the function that mentally rotates the photograph of a body part could be a useful means of reinforcing simple motor imagery. It is conceivable that mental rotation is an embodied cognitive process, involving simulation of the movements of one's own body. Motor imagery training has the effect of improving the motor function of hemiplegics patients; however, the effect of therapeutic intervention on motor imagery is by no means confirmed, because the factors influencing motor imagery are unclear.

The purpose of this research was to measure the mental rotation capacity of hemiplegic patients using photographs of a rotated hand, and to examine the influence of mental rotation on the affected side of stroke patients.

\section{SUBJECTS AND METHODS}

The participants in this study were 31 hospitalized hemiplegic stroke patients (cerebrovascular accident [CVA] group, $64.5 \pm 9.5$ years old) and 29 healthy individuals living in the community (control [CTL] group, $63.5 \pm 11.7$ years old). We excluded CVA patients with severe pain, dementia, aphasia, ataxia, and parkinsonism (according to medical records) (Table 1). Subjects with physical or cognitive impairments were also excluded from the CTL group. 
Table 1. Characteristics of the 31 CVA patients

\begin{tabular}{ccc}
\hline & & CVA \\
\hline Patients (n) & & 31 \\
Right hemiplegia : Left hemiplegia (n) & & $13: 18$ \\
Age (y) & MCA territory & $64.5 \pm 9.5$ \\
& Putamen & 11 \\
& Thalamus & 7 \\
& ACA territory & 4 \\
Elapsed days from onset (d) & Basal ganglia & 4 \\
Motor impairment; SIAS & Brainstem & 2 \\
Sensory impairment; FMA & Upper limb & $46.3 \pm 24.8$ \\
Impaired attention; ARS & Upper limb & $3.5 \pm 1.7 / 5$ \\
Reaction time (s) & & $3.2 \pm 1.0 / 4$ \\
& Upper limb on affected side & $14.5 \pm 10.2 / 56$ \\
& Upper limb on unaffected side & $2.5 \pm 0.9$ \\
\end{tabular}

Mean \pm SD. CVA, persons who sustained a cerebrovascular accident; MCA, middle cerebral artery; ACA, anterior middle cerebral artery; SIAS, Stroke Impairment Assessment Set; FMA, Fugl-Meyer Assessment; ARS, Attentional Rating Scale.

All subjects gave their informed, written consent to participation in the study. This study used a cohort study design.

Mental rotation by the participants was measured using a personal computer. Photographs depicting the dorsal and plantar views of the right and left hands were displayed on a screen. Each photograph was then randomly rotated clockwise to $0^{\circ}, 90^{\circ},-90^{\circ}$, and $180^{\circ}$. Sixteen photographs were displayed in random order. The subjects viewed the photographs and were asked to identify the pictures as quickly as possible by using a fingertip to push a button to indicate "right hand" or "left hand". The subject's reaction time for mental rotation was recorded. Moving a hand or looking at hand was not allowed at that time. The photograph was displayed until the subject answered, which was defined as the reaction time (seconds). Measurements were performed after one of the demonstration, and the mean value of the reaction time was calculated.

The Attentional Rating Scale [ARS] was used to assess attention in stroke patients. ARS is a method of evaluating impaired attention in $\mathrm{ADL}^{14)}$ and classifies impaired attention into five scales. It is composed of 14 items assessed on a scale of 0 (none) to 4 (always), with a sum of 56 points. Individuals with high total points were judged as having a serious impairment.

The Stroke Impairment Assessment Set [SIAS] was used to evaluate motor function of the upper limbs ${ }^{15}$. The SIAS assesses various aspects of impairment in hemiplegic patients. Motor function of the upper limb and fingers on the affected side was evaluated in six stages on a scale of 0 (none) to 5 (normal). After evaluation, the median value of the SIAS scale of the upper limb and fingers was calculated.

Fugl-Meyer Assessment [FMA] was used for deep sensory measurements of the upper limb ${ }^{16)}$. FMA is an international evaluation method of physical function in stroke patients. Deep sensory measurements of the upper limb were classified into five stages on a scale of 0 (anesthesia) to 4 (normal) at the shoulder, elbow and finger on the affected side. Following the measurement, the mean value of the FMA scale of the shoulder, elbow, and finger was calculated.

The reaction time was measured on the affected side in CVA, and the unaffected side in the CVA and CTL groups. Data were analyzed with $3 \times 4$ (group $\times$ angle) two-way analysis of variance [two-way ANOVA] with repeated measures for one variable and appropriate post-hoc analysis. In addition, ANOVA followed by Tukey's post-hoc test was used to compare subjects' reaction times. The reaction time for each rotation angle on the affected side was compared in the CVA group. The reaction time on the affected side was evaluated to identify influential factors from among lapsed days from the onset of stroke, the brain-damaged side, attention, and motor and sensory functions by multiple regression analysis (stepwise method). $\mathrm{p}<0.05$ was considered significant.

\section{RESULTS}

The reaction times were $1.7 \pm 0.8 \mathrm{~s}$ in the CTL group, 2.5 $\pm 0.9 \mathrm{~s}$ on the affected side in the CVA group, and $2.2 \pm 0.7$ $\mathrm{s}$ on the unaffected side in the CVA group (Table 2).

Two-way ANOVA showed that the physical function and rotation angle of the photograph had a significant effect on the reaction time $(\mathrm{p}<0.001)$, with no interaction between them (Table 2). This result indicates that the reaction time is associated with the physical impairment and the rotation angle of the photograph.

ANOVA showed significant differences in the reaction time between the CVA and CTL groups $(p<0.01)$. Further statistical post-hoc analysis of the data showed that the reaction time on the affected side in the CVA group was significantly delayed in comparison with the CTL group $(p<0.05)$; however, there were no differences in the reaction times between the unaffected side in the CVA and CTL groups, and between the affected side and unaffected side in the CVA group (Table 2).

In addition, ANOVA showed differences in the reaction time of each rotation angle in the CVA group $(p<0.01)$. 
Table 2. Reaction times of the CVA and CTL groups at each rotation angle

\begin{tabular}{lccc}
\hline & \multicolumn{2}{c}{ CVA group } & CTL group \\
& Affected side & Unaffected side & \\
\hline $0^{\circ}$ & $2.0 \pm 0.7^{*}$ & $2.1 \pm 1.0$ & $1.6 \pm 0.8$ \\
$90^{\circ}$ & $2.2 \pm 1.0^{*}$ & $2.0 \pm 1.0$ & $1.5 \pm 0.7$ \\
$-90^{\circ}$ & $2.4 \pm 1.3^{*}$ & $2.2 \pm 1.1$ & $1.7 \pm 0.8$ \\
$180^{\circ}$ & $3.0 \pm 1.8^{*}, \#$ & $2.6 \pm 1.2$ & $2.0 \pm 1.3$ \\
Total & $2.5 \pm 0.9^{*}$ & $2.2 \pm 0.7$ & $1.7 \pm 0.8$ \\
\hline
\end{tabular}

Score: seconds. Mean $\pm \mathrm{SD} .{ }^{*} \mathrm{p}<0.01$, compared with CTL group. ${ }^{\#} \mathrm{p}<0.01$, compared with a rotation $0^{\circ}$ on the affected side. CVA, persons who sustained a

Table 3. Multiple regression analysis of the CVA group reaction times

\begin{tabular}{lccc}
\hline & \multicolumn{3}{c}{ Objective variable: Reaction time on affected side } \\
& $\mathrm{R}^{2}=0.236, \mathrm{p}=0.006$ (Stepwise method, Step-down procedure) \\
\hline Regression coefficient & Standard error & Standardized partial regression coefficient \\
\hline Impaired attention & 0.044 & 0.015 & 0.486 \\
\hline
\end{tabular}

CVA, persons who sustained a cerebrovascular accident.

Statistical post-hoc analysis of the data showed that the reaction time for the affected side to a photograph rotated $180^{\circ}$ was delayed in comparison with that to a photograph rotated in the CVA group $(\mathrm{p}<0.01)$.

Multiple regression analysis (stepwise method, $\mathrm{R}^{2}=0.236, \mathrm{p}=0.006, \beta=0.486$ ) disclosed that the reaction time on the affected side in the CVA group was affected by impaired attention (Table 3).

\section{DISCUSSION}

Mental rotation is performed with the direction of the hand in a photograph coinciding with that of one's own hand, and is used to identify whether the photograph depicts the right or left hand. It is considered that the reaction time reflects motor imagery capacity ${ }^{17}$ ). Our results showed that the impaired ability of motor imagery in stroke patients was less when viewing photographs on the affected side than on the unaffected side. This result is consistent with a previous report on the impaired ability of motor imagery in stroke patients ${ }^{18)}$. The main finding of the present study was that attention disturbances are associated with mental rotation ability; by contrast, those of motor function, deep sensory factor, lapsed days from onset, and brain-damaged side, were less associated with mental rotation ability in the CVA group. These findings are not consistent with previous reports that physical characteristics are associated with the reaction time of mental rotation ${ }^{10,12,13,18)}$. It is considered that the mental rotation capacity is low when there is a difference between recognition of self-motion and the actual motion in brain-damaged patients who have impaired attention. Hence, our results indicate that motor imagery dose not generate motor plans that depend on the current configuration of the limbs in stroke patients, suggesting that stroke patients view photographs of mental rotation as visual imagery without kinesthetic imagery.

In rehabilitation-related research, some studies have reported that physical function is improved by mental practice in stroke patients ${ }^{2-6,19)}$; however, our results showed that impaired motor imagery ability in the CVA group, suggesting that a strategy for therapeutic intervention with motor imagery is expected to have a high therapeutic effect because of the impaired attention of stroke patients.

In the present study, the number of participants was too few to test reliability. More participants are required to evaluate mental rotation to improve its use as a therapeutic intervention, and objective assessment was lacking in the evaluation of mental rotation. We think that to measure mental rotation, it is necessary to evaluate the details of the brain activity, using a functional brain imaging device such as functional magnetic resonance imaging. The reaction time for mental rotation of stroke patients with attention disturbances within three months from onset is impaired; however, what would be the outcome in stroke patients in the chronic stage. Whether an intervention of motor imagery training is appropriate for stroke patients is an issue for further discussion.

\section{REFERENCES}

1) Alberts MJ, Ovbiagele B: Current strategies for ischemic stroke prevention: Role of multimodal combination therapies. J Neurol, 2007, 254: 1414-1426.

2) Malouin F, Belleville S, Richards CL, et al.: Working memory and mental practice outcomes after stroke. Arch Phys Med Rehabil, 2004, 85: 177-183.

3) Page SJ, Levine P, Leonard AC: Effects of mental practice on affected limb use function in chronic stroke. Arch Phys Med Rehabil, 2005, 86: 399-402.

4) Butler AJ, Page SJ: Mental practice with motor imagery: evidence for motor recovery and cortical reorganization after stroke. Arch Phys Med Rehabil, 2006, 87: S2-S11.

5) Dickstein R, Deutsch JE: Motor imagery in physical therapist practice. Physical Therapy, 2007, 87: 942-953.

6) Page SJ, Levine P, Leonard A: Mental practice in chronic Stroke: results of a randomized, placebo-controlled trial. Stroke, 2007, 38: 1293-1297.

7) Decety J, Grèzes J: Neural mechanisms subserving the perception of human actions. Trends Cogn Sci, 1999, 3: 172-178.

8) Jackson PL, Lafleur MF, Malouin F, et al.: Potential role of mental practice using motor imagery in neurologic rehabilitation. Arch Phys Med Rehabil, 2001, 82: 1133-1141.

9) Michelon P, Vettel JM, Zacks JM: Lateral somatotopic organization during 
imagined and prepared movements. J Neurophysiol, 2006, 95: 811-822.

10) Parsons LM: Integrating cognitive psychology, neurology and neuroimaging. Acta Psychol, 2001, 107: 155-181.

11) Richter $W$, Somorjai $R$, Summers $R$, et al.: Motor area activity during mental rotation studied by time-resolved single trial fMRI. J Cogn Neurosci, 2000, 12: $310-320$.

12) Vingerhoets G, de Lange FP, Vandemaele P: Motor imagery in mental rotation: an fMRI study. Neuroimage, 2002, 17: 1623-1633.

13) de Lange FP, Helmich PC, Toni I: Posture influences motor imagery: an fMRI study. Neuroimage, 2006, 33: 609-617.

14) Ponsford JL, Kinsella G: Evaluation of a remedial programme for attentional deficits following closed-head injury. J Clin Exp Neuropsychol, 1988, 6: 693-708.
15) Tsuji T, Liu M, Sonoda S, et al.: The Stroke impairment assessment set: its internal consistency and predictive validity. Arch Phys Med Rehabil, 2000, 81: 863-868.

16) Fugl-Meyer AR, Jaasko L, Leyman I, et al.: The post-Stroke hemiplegic patient. 1. a method for evaluation of physical performance. Scand J Rehabil Med, 1975, 7: 13-31.

17) Moseley GL: Why do people with complex regional pain syndrome take longer to recognize their affected hand? Neurology, 2004, 62: 2182-2186.

18) Shenton JT, Schwoebel J, Coslett HB: Mental motor imagery and the body schema: evidence for proprioceptive dominance. Neurosci Lett, 2004, 370: 19-24.

19) Molder T: Motor imagery and action observation: cognitive tool for rehabilitation. J Neural Trans, 2007, 114: 1265-1278. 